\title{
MicroRNA-106b regulates skeletal muscle insulin sensitivity and glucose homeostasis by targeting mitofusion-2
}

\author{
YING ZHANG $^{1,2}$, WEI HE ${ }^{1}$, YUAN-FU GAO ${ }^{1}$, ZHONG-MIN FAN ${ }^{1}$, CHUN-LIN GAO $^{1}$ and ZHENG-KUN XIA ${ }^{1}$ \\ ${ }^{1}$ Department of Pediatrics, Jinling Hospital, Nanjing University School of Medicine, Nanjing, \\ Jiangsu 210002; ${ }^{2}$ School of Medicine, Nankai University, Tianjin 300071, P.R. China
}

Received October 11, 2015; Accepted August 30, 2016

DOI: $10.3892 / \mathrm{mmr} .2017 .7439$

\begin{abstract}
MicroRNA-106b (miR-106b) is reported to be closely associated with skeletal muscle insulin resistance. The present study further investigated the role of miR-106b in skeletal muscle insulin sensitivity and glucose homeostasis in vivo. Mice were randomly divided into 4 groups and infected with lentivirus expressing miR-106b (miR-106b mice), miR-106b sponge (miR-106b inhibition mice) or the corresponding empty vectors. Mitofusion-2 (Mfn2) protein expression levels and glucose transporter (Glut)-4 protein translocation were significantly reduced in the muscle of miR-106b mice, whereas they were unaffected in miR-106b inhibition mice. miR-106b mice had significantly increased blood glucose levels following $12 \mathrm{~h}$ of fasting and impaired glucose tolerance, whereas miR-106b inhibition mice had no significant alterations in fasting blood glucose levels and glucose tolerance. In vitro, the suppressive effect of miR-106b on glucose uptake and Glut 4 translocation was completely inhibited in $\mathrm{C} 2 \mathrm{C} 12$ myotubes infected with Mfn2 plasmids. Following treatment of $\mathrm{C} 2 \mathrm{C} 12$ myotubes with Mfn2 small interfering RNA, miR-106b inhibition consistently increased Mfn2 protein levels and improved glucose uptake and Glut4 translocation. These results indicated that miR-106b targeted Mfn2 and regulated skeletal muscle insulin sensitivity and glucose tolerance. Therefore, increased miR-106b expression may be a potential mechanism underlying insulin resistance and type 2 diabetes.
\end{abstract}

\section{Introduction}

Type 2 diabetes mellitus (T2DM) is a primary health concern, reaching epidemic proportions worldwide. The International

Correspondence to: Dr Chun-Lin Gao or Dr Zheng-Kun Xia, Department of Pediatrics, Jinling Hospital, Nanjing University School of Medicine, 305 Zhongshan East Road, Nanjing, Jiangsu 210002, P.R. China

E-mail: shuangmu34@163.com

E-mail:njxzk@126.com

Key words: microRNA-106b, mitofusion-2, skeletal muscle insulin resistance, glucose homeostasis
Diabetes Federation has predicted that the number of patients with diabetes will increase from 240 million in 2007 to 380 million in 2025 (1). Current treatments for T2DM involve attenuating the increased glucose level by insulin, or by agents which increase the sensitivity of tissue to insulin, or inhibit glucose absorption by small intestine. Useful therapeutic targets for T2DM remain to be identified. Resistance to the pleiotropic effects of insulin in the primary insulin target organs is a critical process in the development of the disease; however, the molecular mechanisms underlying insulin resistance remain to be fully elucidated. MicroRNAs (miRNAs) are a class of small, non-coding RNAs widely expressed in all multicellular organisms that post-transcriptionally regulate gene expression (2). Previous studies have highlighted the significance of miRNAs in maintaining metabolic homeostasis. miR-130-3p and miR-108a, for example, have been demonstrated to regulate insulin sensitivity $(3,4)$.

Muscle tissue is an important site of postprandial glucose uptake; $75 \%$ of insulin-dependent glucose removal from the plasma takes place in the skeletal muscle. Therefore, muscle is critical for systemic glucose homeostasis. miR-24 and miR-126 contribute to the adaptation of muscle tissue to increased glucose levels; however, they do not appear to be involved in the pathogenesis of insulin resistance (5). Previous studies have demonstrated that miR-106b is highly expressed in the skeletal muscle of diabetes patients (6) and insulin resistant mice (7). Our previous study revealed that miR-106b induced mitochondrial dysfunction and insulin resistance in $\mathrm{C} 2 \mathrm{C} 12$ myotubes by targeting mitofusin-2 (Mfn2) (8); silencing miR-106b improved the tumor necrosis factor (TNF)- $\alpha$ - and palmitic acid (PA)-induced insulin resistance and mitochondrial dysfunction in these cells $(8,9)$. The present study aimed to further investigate the contribution of miR-106b to skeletal muscle insulin sensitivity and glucose homeostasis in vivo and discuss the role of Mfn2 in miR-106b-induced insulin resistance in vitro.

\section{Materials and methods}

Plasmids and lentiviruses construction and packaging. Lentiviruses with miR-106b for overexpression and miR-106b sponge for downregulation were constructed and packaged as previously described (8). The pre-miR-106b fragment was cloned from the genomic DNA of mouse skeletal muscle 
tissue. The miR-106b inhibitor sponge was synthesized by Shanghai GenePharma Co., Ltd. (Shanghai, China). Plasmids of miR-106b and miR-106b sponge were inserted into the lentiviral vector pGIPZ (Invitrogen; Thermo Fisher Scientific, Inc., Waltham, MA, USA). The DNA fragment encoding Mfn2 was amplified using genomic DNA of mouse skeletal muscle as the polymerase chain reaction (PCR) template and inserted into the expression vector pc3.1-puro (Invitrogen; Thermo Fisher Scientific, Inc.).

Animals and treatment. A total of 24 male C57BL/6J mice (age, 6-8 weeks) were obtained from the Laboratory Animal Center of Jinling Hospital (Nanjing, China). Mice were housed under a 12-h light/dark cycle at $25^{\circ} \mathrm{C}$ with ad libitum access to commercial rodent chow and tap water prior to the initiation of the experiments. The present study was approved by, and conducted in accordance with the guidelines of, the Institutional Animal Care and Use Committee of Nanjing General Hospital of Nanjing Military Command (Nanjing, China). Mice were randomly divided into 4 groups and infected with lentivirus expressing miR-106b $(n=6)$ or miR-106b sponge $(n=6)$ and corresponding empty vectors ( $\mathrm{n}=6$ in each group). Briefly, $1 \times 10^{7}$ transduction units of lentivirus in phosphate-buffered saline at a volume of $10 \mathrm{ml} / \mathrm{kg}$ were intravenously injected into the tail vein of mice one week prior to a glucose tolerance test (GTT). Blood glucose levels were measured using an Elite glucometer (Bayer AG, Leverkusen, Germany). GTT was performed by intraperitoneal injection of $2 \mathrm{~g} / \mathrm{kg}$ glucose following $12 \mathrm{~h}$ of fasting. Blood glucose levels were measured 15, 60 and 90 min following GTT. Subsequently, mice were anesthetized by an intraperitoneal injection of $1 \%$ sodium pentobarbital (40 mg/kg; Invitrogen; Thermo Fisher Scientific, Inc.). The quadricep muscles were collected following perfusion with saline.

Cell culture and treatment. C2C12 mouse myoblasts (American Type Culture Collection, Manassas, VA, USA) were cultured at $37^{\circ} \mathrm{C}$ in Dulbecco's modified Eagle's medium (DMEM) containing $10 \%$ fetal bovine serum (Wisent, Inc., St. Bruno, QC, Canada). C2C12 myoblasts seeded in 12-well plates $\left(4 \times 10^{4}\right.$ cells/well) were transfected with the aforementioned Mfn2 plasmid (1.6 $\mu \mathrm{g} /$ well), $20 \mu \mathrm{M}$ Mfn2 small interfering (si)RNA or control siRNA (Santa Cruz Biotechnology, Inc., Dallas, TX, USA), and lentiviruses with miR-106b or miR-106b sponge. The stable C2C12 cells lines with miR-106b overexpression and downregulation were obtained by selecting with puromycin for two weeks. When $\mathrm{C} 2 \mathrm{C} 12$ myoblasts reached confluence, the medium was replaced with differentiation medium containing DMEM and 2\% horse serum (Gibco; Thermo Fisher Scientific, Inc.), which was replaced every other day. Following five additional days, the differentiated C2C12 myoblasts had fused into myotubes.

2-Deoxyglucose uptake assay. The uptake of 2-deoxy- $D-\left[{ }^{3} \mathrm{H}\right]$ glucose (Beijing CIC Technology Co., Ltd., Beijing, China) was assayed as previously described (8). Following $4 \mathrm{~h}$ of serum starvation in DMEM, C2C12 myotubes were rinsed twice with 4-(2-hydroxyethyl)-1-piperazineethanesulfonic acid (HEPES)-buffered saline (20 mM HEPES, $140 \mathrm{mM}$ $\mathrm{NaCl}, 5 \mathrm{mM} \mathrm{KCl}, 2.5 \mathrm{mM} \mathrm{MgSO}_{4}, 1 \mathrm{mM} \mathrm{CaCl}_{2}$; pH 7.4), and stimulated with $100 \mathrm{nM}$ insulin (Peptide Institute, Inc., Osaka, Japan) for $30 \mathrm{~min}$ at $37^{\circ} \mathrm{C}$. Glucose uptake was assessed by the addition of $10 \mu \mathrm{M} 2$-deoxyglucose containing $0.2 \mu \mathrm{Ci}$ $\left[{ }^{3} \mathrm{H}\right]$-2-deoxyglucose in HEPES-buffered saline for $10 \mathrm{~min}$. The uptake of $\left[{ }^{3} \mathrm{H}\right]$-2-deoxyglucose was terminated by ice-cold phosphate-buffered saline (PBS; $8 \%$ glucose), and the cells were immediately washed with ice-cold PBS. Subsequently, cells were lysed with $0.25 \mathrm{~N} \mathrm{NaOH}$ and the cell lysates were transferred to scintillation vials (Beckman Coulter, Inc., Brea, CA, USA) to measure the radioactivity using an LS6500 liquid scintillation counter (Beckman Coulter).

Reverse transcription-quantitative polymerase chain reaction (RT-qPCR). miRNAs were extracted using an miRNeasy Mini kit (Qiagen China Co., Ltd., Shanghai, China). miR-106b cDNA was generated with a RT primer provided with the TaqMan ${ }^{\circledR}$ MicroRNA Reverse Transcription kit (Thermo Fisher Scientific, Inc.). qPCR was performed on an ABI7500 RT-PCR system using TaqMan Gene Expression assays for miR-106b (Mm03306675_pri; Thermo Fisher Scientific, Inc.) and U6 (Mm01164115_g1; Thermo Fisher Scientific, Inc.). The cycling conditions were as follows: Initial denaturation for $10 \mathrm{~min}$ at $95^{\circ} \mathrm{C}$, followed by 40 cycles of $15 \mathrm{sec}$ of denaturation at $95^{\circ} \mathrm{C}, 30 \mathrm{sec}$ annealing at the optimal primer temperature and $36 \mathrm{sec}$ extension at $72^{\circ} \mathrm{C}$. Each sample was assayed in duplicate. Negative controls (no template) were run to ensure the absence of contamination. Analysis was performed using the $2^{-\Delta \Delta \mathrm{Cq}}$ method (10) and the data was normalized to U6.

Western blot analysis. Western blot analysis was performed as previously described (8). Cells and tissue were lysed with radioimmunoprecipitation assay lysis buffer (Beyotime Institute of Biotechnology, Haimen, China) for $20 \mathrm{~min}$ on ice. Protein levels were quantified using a bicinchoninic acid protein assay kit (Pierce; Thermo Fisher Scientific, Inc.) according to the manufacturer's protocol. Proteins from muscle tissue $(60 \mu \mathrm{g})$ or cells $(30 \mu \mathrm{g})$ were loaded onto $10 \%$ SDS-PAGE gels. Following electrophoresis, proteins were transferred to nitrocellulose membranes (EMD Millipore, Billerica, MA, USA). Membranes were blocked with 5\% bovine serum albumin (BSA) in Tris-buffered saline with Tween-20 [TBST; 50 mM Tris (pH 7.5), $150 \mathrm{mM} \mathrm{NaCl,} \mathrm{0.05 \%}$ Tween-20] for $12 \mathrm{~h}$. Following this, membranes were incubated at $4^{\circ} \mathrm{C}$ overnight with one of the following primary antibodies at the indicated dilutions in TBS with 5\% BSA: Rabbit anti-Mfn2 (1:500; catalog no. ab50838), rabbit anti-glucose transporter-4 (Glut4; 1:500; catalog no. ab654), and rabbit anti- $\beta$-actin (1:5,000; catalog no. ab8227), purchased from Abcam (Cambridge, MA, USA). The membranes were washed five times for $5 \mathrm{~min}$ with TBST. Subsequently, the membranes were incubated with a horseradish peroxidase-conjugated goat anti-rabbit IgG (1:5,000; catalog no. ab6721; Abcam) for $1 \mathrm{~h}$ at room temperature, washed with TBST and developed using an Enhanced Chemiluminescence kit (GE Healthcare Life Sciences, Chalfont, UK). Densitometry was performed using Quantity One software version 4.6.4 (Bio-Rad Laboratories, Inc., Hercules, CA, USA).

Statistical analysis. Data are expressed as the mean \pm standard deviation. Differences between groups were assessed by 

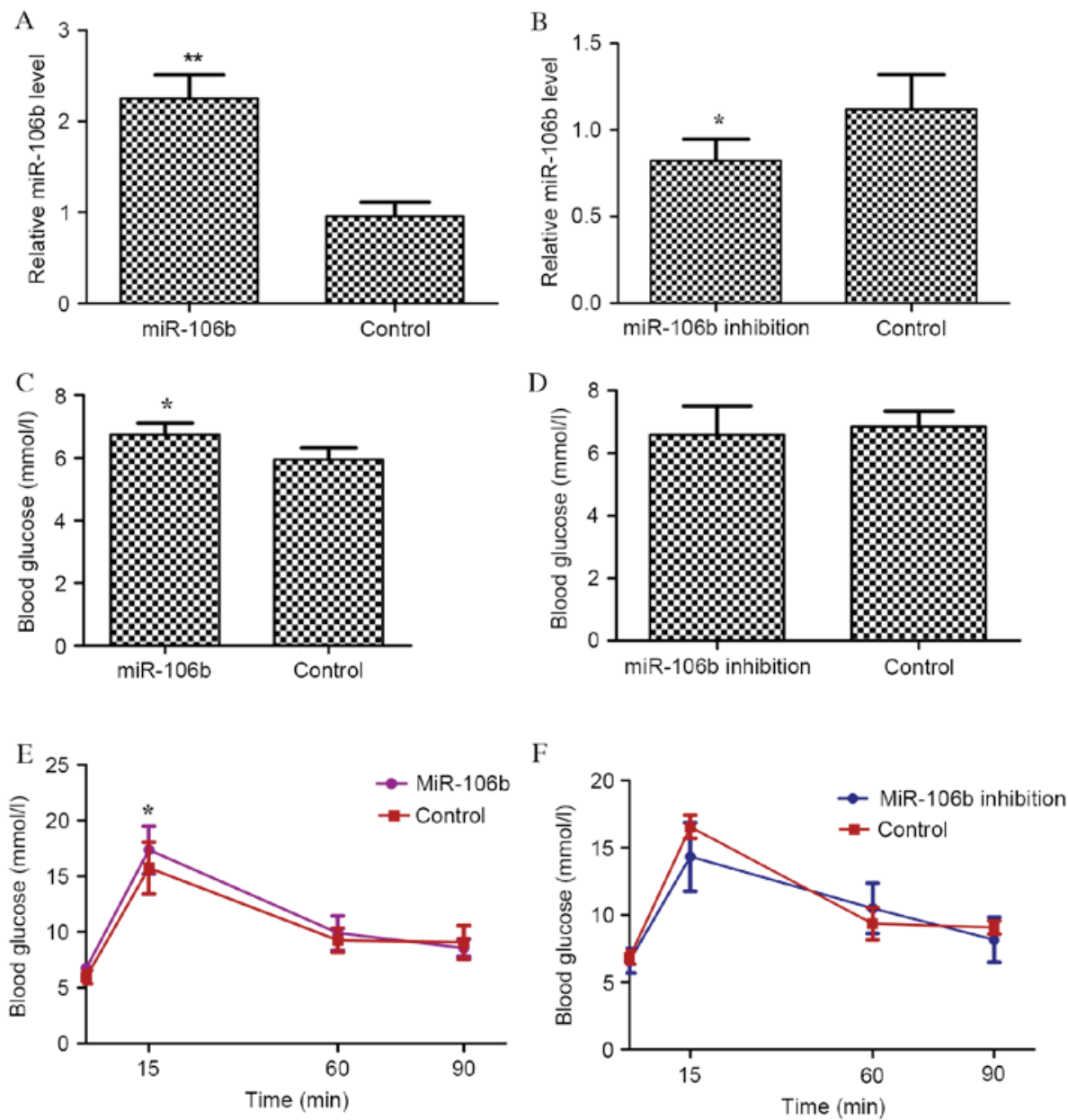

Figure 1. miR-106b regulates skeletal muscle insulin sensitivity and glucose homeostasis in vivo. miR-106b expression levels in the muscle of mice injected with (A) lentivirus with miR-106b for overexpression (miR-106b mice) or (B) miR-106b sponge for downregulation (miR-106b inhibition mice). (C) miR-106b increased the 16-h fasting blood glucose level in miR-106b mice, (D) but this level was unaffected in miR-106b inhibition mice. (E) miR-106b affected blood glucose tolerance in miR-106b mice, (F) but blood glucose tolerance was not altered in miR-106b inhibition mice. ${ }^{*} \mathrm{P}<0.05$ and ${ }^{* *} \mathrm{P}<0.01$ vs. control. miR, microRNA.

two-tailed Student's $t$-test or one-way analysis of variance followed by the Student-Newman-Keuls test. $\mathrm{P}<0.05$ was considered to indicate a statistically significant difference.

\section{Results}

miR-106b regulates skeletal muscle insulin sensitivity and glucose homeostasis in vivo. To investigate whether miR-106b regulated glucose homeostasis in vivo, lentiviruses with miR-106b for overexpression, with miR-106b sponge for downregulation or empty lentiviral vectors were used to infect C57BL/6 mice by tail vein injection. The expression level of miR-106b was markedly up and downregulated in the muscle of mice infected with lentiviruses expressing miR-106b precursors (designated as miR-106b mice; $\mathrm{P}=0.009$; Fig. 1A) and miR-106b sponge (designated as miR-106b inhibition mice; $\mathrm{P}=0.007$; Fig. 1B), respectively. miR-106b mice had significantly greater blood glucose levels following $16 \mathrm{~h}$ of fasting ( $\mathrm{P}=0.005$; Fig. 1C), whereas miR-106b inhibition mice had no significant alteration in blood glucose (Fig. 1D). Similarly, miR-106b mice had impaired glucose tolerance $(\mathrm{P}<0.001$; Fig. 1E), whereas miR-106b inhibition mice did not (Fig. 1F).
Mfn2 ( $\mathrm{P}=0.027$; Fig. 2A) and plasmalemma Glut4 ( $\mathrm{P}=0.034$; Fig. 2B) protein expression levels were significantly reduced in the muscle of miR-106b mice, whereas these protein levels were unaffected in miR-106b inhibition mice. The decrease of Glut4 translocation to cell membrane in the muscle indicated reduced insulin sensitivity.

$M f n 2$ is involved in the regulation of C2C12 myotubes insulin sensitivity by miR-106b in vitro. To investigate whether Mfn2 is involved in the regulation of muscle insulin sensitivity by miR-106b, C2C12 myotubes were transfected with miR-106b, miR-106b sponge, Mfn2 plasmid or Mfn2 siRNA. miR-106b successfully decreased insulin-stimulated glucose uptake in C2C12 myotubes overexpressing miR-106b (pGIPZ-MiR; $\mathrm{P}=0.042$; Fig. 3A). However, the repressive effect of miR-106b on glucose uptake was completely inhibited in $\mathrm{C} 2 \mathrm{C} 12$ myoblasts with miR-106b overexpression and Mfn2 plasmid infection (Pc3.1-Mfn2; $\mathrm{P}=0.032$ ), but not by control plasmid (Pc3.1). Mfn2 protein expression levels $(\mathrm{P}=0.043)$ and translocation of Glut4 $(\mathrm{P}=0.034)$ were significantly decreased in C2C12 myotubes with miR-106b overexpression; however, Mfn2 plasmid infection significantly increased Mfn2 
A

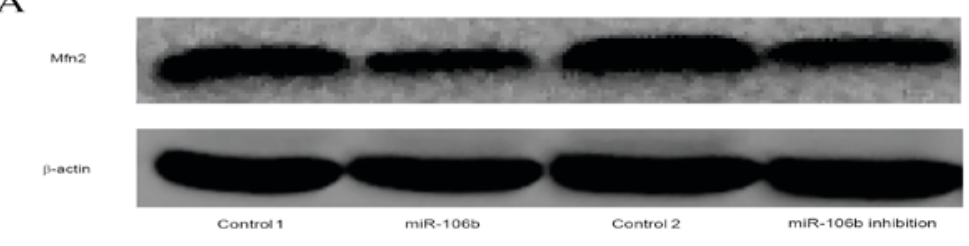

B

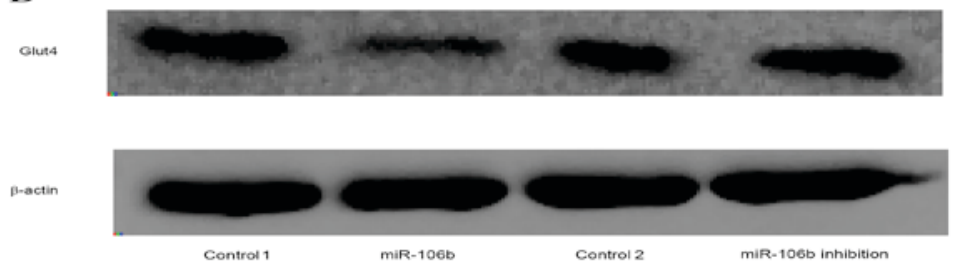

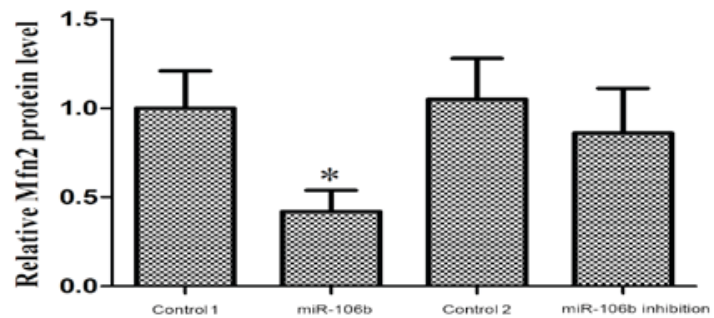

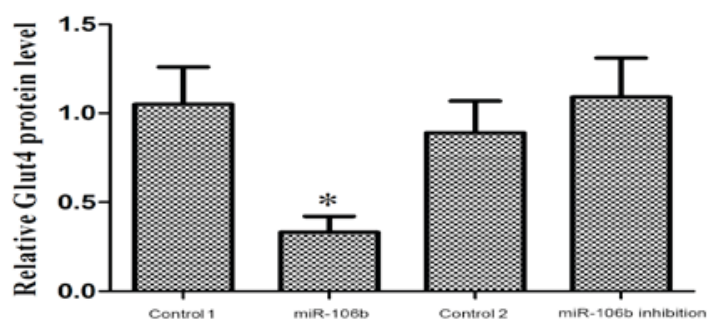

Figure 2. miR-106b regulates Mfn2 and Glut4 protein expression levels. (A) Mfn2 protein expression levels in the skeletal muscle of mice were decreased in miR-106b mice, but not in miR-106b inhibition mice. (B) Glut4 protein expression levels in the skeletal muscle of mice were decreased in miR-106b mice, but not in miR-106b inhibition mice. ${ }^{*} \mathrm{P}<0.05$ vs. control 1. miR, microRNA; Mfn2, mitofusin-2; Glut4, glucose transporter-4.

$(\mathrm{P}=0.023)$ and Glut4 protein expression levels $(\mathrm{P}=0.011)$ in $\mathrm{C} 2 \mathrm{C} 12$ myotubes with miR-106b overexpression compared with control plasmids (Fig. 3B and C).

Inhibition of miR-106b (pGIPZ-spon) did not significantly affect glucose uptake in $\mathrm{C} 2 \mathrm{C} 12$ myotubes infected with control siRNA [siRNA-no target (NT); $\mathrm{P}=0.322$ ]. $\mathrm{C} 2 \mathrm{C} 12$ myotubes treated with Mfn2 siRNA (siRNA-Mfn2) had decreased glucose uptake $(\mathrm{P}=0.012)$; however, this was increased by the concomitant inhibition of miR-106b ( $\mathrm{P}=0.032$; Fig. 4A). Glut4 translocation was unaffected by inhibition of miR-106b $(\mathrm{P}=0.123)$; however, Mfn2 protein expression levels were significantly increased in $\mathrm{C} 2 \mathrm{C} 12$ myotubes with miR-106b inhibition ( $\mathrm{P}<0.001$; Fig. $4 \mathrm{~B}$ and $\mathrm{C})$. Mfn2 siRNA infection significantly decreased Mfn2 expression levels and Glut4 translocation in $\mathrm{C} 2 \mathrm{C} 12$ myotubes; miR-106b inhibition partially reversed these effects. These results indicated that miR-106b regulated skeletal muscle insulin sensitivity by targeting Mfn2.

\section{Discussion}

Gallagher et al (6) identified, using an miRNA microarray, that miR-106b was highly expressed in the skeletal muscle of T2DM patients. A separate study revealed, using microarrays and RT-qPCR, that miR-106b expression in the skeletal muscle of mice fed a high-fat diet was 4.19-fold greater than mice on a normal diet (7). However, the role of miR-106b in regulating skeletal muscle insulin sensitivity and glucose homeostasis remains to be fully elucidated.

In our previous study, miR-106b overexpression was demonstrated to decrease glucose uptake and Glut4 translocation in $\mathrm{C} 2 \mathrm{C} 12$ myotubes, indicating insulin resistance (8). In addition, it was revealed that miR-106b targeted Mfn2, an important protein mediating mitochondrial fusion and dynamics, inducing mitochondrial dysfunction, which may contribute to muscle insulin resistance (8). The inflammatory factor TNF- $\alpha$ and the saturated fatty acid PA increased miR-106b expression in $\mathrm{C} 2 \mathrm{C} 12$ myotubes in a time- and dose-dependent manner $(7,8)$. miR-106b loss of function attenuated TNF- $\alpha$ - and PA-induced mitochondrial dysfunction and insulin resistance $(8,9)$.

In the present study miR-106b mice and miR-106b inhibition mice were created to increase or reduce miR-106b expression levels in the muscle, through tail-vein injection of lentiviruses with miR-106b for overexpression or miR-106b sponge for downregulation. miR-106b mice had decreased Mfn2 and Glut4 expression levels in the muscle, and impaired glucose tolerance at the first phase by GTT. miR-106b inhibition failed to regulate systemic glucose homeostasis in vivo. This may be due to the decrease of glucose uptake by the muscle being compensated for by the increase of glucose uptake by other insulin target organs, including the liver and adipose tissue. Furthermore, energy metabolism is a complex and integrated process, and gluconeogenesis and glycolysis additionally contribute to systemic glucose homeostasis. The present study indicated that miR-106b contributed to glucose homeostasis in certain pathological processes.

In our previous study, it was demonstrated that Mfn2 was a direct target of miR-106b (8). In the present study, it was further revealed, by overexpression or knockdown of Mfn2 expression in $\mathrm{C} 2 \mathrm{C} 12$ myotubes, that Mfn2 was the direct target through which miR-106b exerted its effects. Mfn2 protein, a dynamin-related protein with GTPase activity, is anchored in the external mitochondrial membrane to mediate mitochondrial fusion (11). In addition, Mfn2 localizes to the endoplasmic reticulum (ER) membrane, serving to tether the ER to mitochondria (12). Mfn2 is highly expressed in skeletal muscle, contributes to the maintenance of mitochondrial morphology and regulates mitochondrial metabolism and intracellular signaling (13). Mfn2 suppression has been detected in skeletal muscle of T2DM patients (14). In addition, a positive correlation between Mfn2 expression in skeletal muscle and insulin 
A

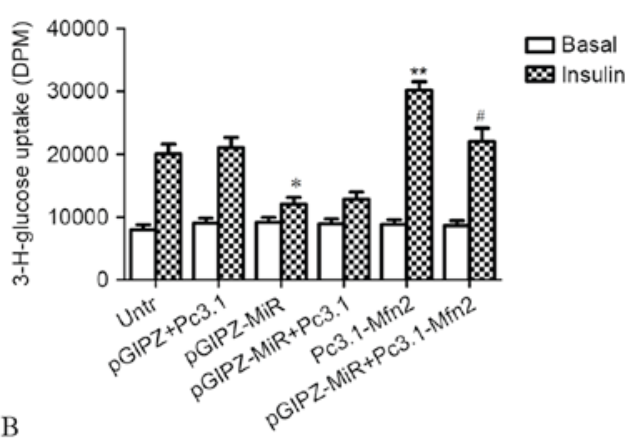

B

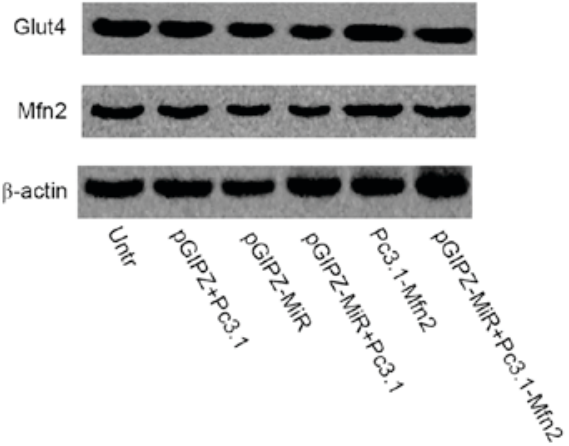

C

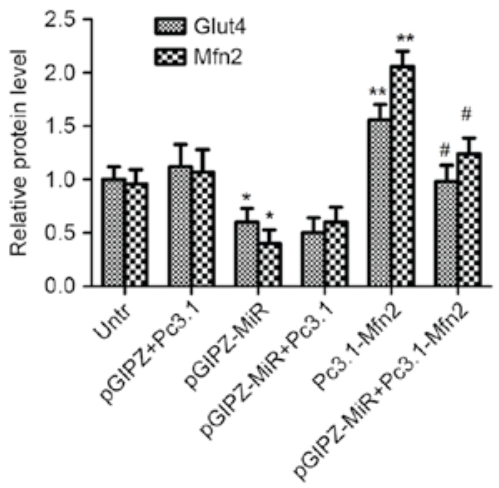

Figure 3. Mfn2 inhibits the miR-106b overexpression-induced decrease of glucose uptake, Mfn2 expression and Glut4 translocation in $\mathrm{C} 2 \mathrm{C} 12$ myotubes. (A) Mfn2 inhibited miR-106b overexpression-induced decrease of insulin-stimulated glucose uptake in $\mathrm{C} 2 \mathrm{C} 12$ myotubes. (B) Western blots of Mfn2 and Glut4 protein expression levels in $\mathrm{C} 2 \mathrm{C} 12$ myotubes. (C) Quantification of western blotting revealed that Mfn2 inhibited miR-106b overexpression-induced decrease of Mfn2 expression levels and Glut4 translocation. ${ }^{*} \mathrm{P}<0.05$ vs. Untr and pGIPZ+Pc3.1; ${ }^{* *} \mathrm{P}<0.01$ vs. Untr and pGIPZ+Pc3.1; ${ }^{\prime} \mathrm{P}<0.05$ vs. pGIPZ-MiR and pGIPZ-MiR+Pc3.1. miR, microRNA; Mfn2, mitofusin-2; Glut4, glucose transporter-4; Untr, untreated; pGIPZ, miR-106b control vector; pGIPZ-MiR, vector containing miR-106b; Pc3.1, Mfn2 control plasmid; Pc3.1-Mfn2, plasmid containing Mfn2.

sensitivity has been detected, as assessed by glucose disposal rates in healthy controls, obese and T2DM patients (14). Furthermore, this positive correlation has been demonstrated in morbidly obese subjects and bariatric surgery patients $(14,15)$. Recently, it was reported that Mfn2 deficiency induced oxidative stress, which contributed to insulin resistance in skeletal muscle cells (16). Mfn2 reduced the accumulation of lipid intermediates in skeletal muscle and alleviated insulin resistance (17). The offspring of rats fed a high-fat diet had reduced Mfn2 mRNA expression levels at postnatal day 35 and 130 (18).
A
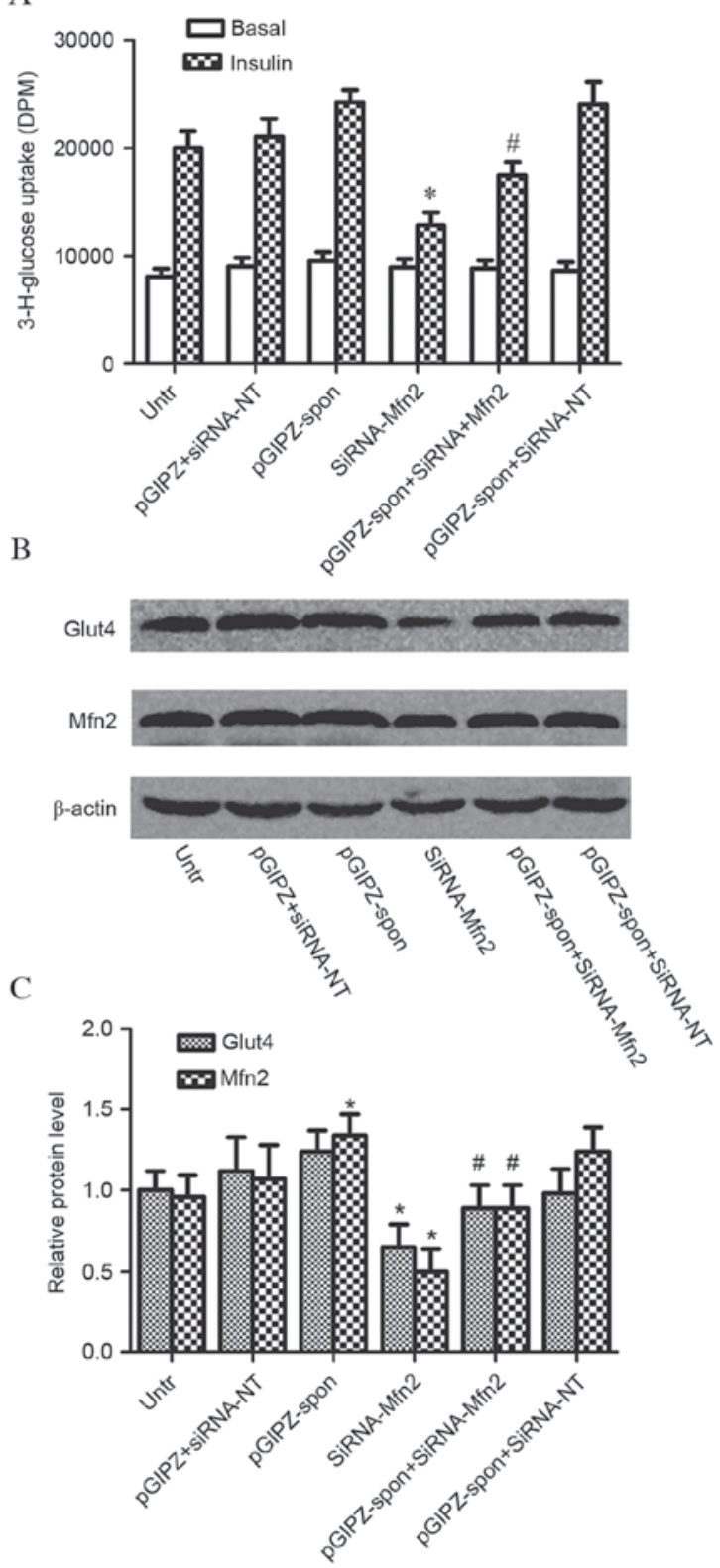

Figure 4. Inhibition of miR-106b consistently improves glucose uptake and Glut4 translocation following Mfn2 downregulation by siRNA. (A) Insulin-stimulated glucose uptake was decreased by Mfn2 siRNA; this decrease was attenuated by inhibition of miR-106b. (B) Western blots of Mfn2 and Glut4 protein expression levels in C2C12 myotubes. (C) Quantification of western blotting revealed that inhibition of miR-106b consistently increased Mfn2 expression and Glut4 translocation following Mfn2 downregulation by siRNA. ${ }^{*} \mathrm{P}<0.05$ vs. Untr and pGIPZ+siRNA-NT; ${ }^{\text {P }}<0.05$ vs. siRNA-Mfn2. miR, microRNA; Mfn2, mitofusin-2; Glut4, glucose transporter-4; Untr, untreated; pGIPZ, miR-106b control vector; pGIPZ-spon, vector containing miR-106b sponge; siRNA, small interfering RNA; siRNA-NT, siRNA no target.

In conclusion, the results of the present study indicated that miR-106b regulated skeletal muscle insulin sensitivity and glucose homeostasis, with Mfn2 contributing to this process. The finding that miR-106b overexpression induced skeletal muscle insulin resistance and impaired glucose homeostasis may suggest a potential mechanism underlying insulin resistance and T2DM. 


\section{Acknowledgements}

The present study was supported by grants from the National Natural Science Foundation of China (grant nos. 81100592 and 81270800).

\section{References}

1. International Diabetes Federation: Diabetes Atlas. 3rd edition. International Diabetes Federation, Brussels, Belgium, 2006.

2. Guo H, Ingolia NT, Weissman JS and Bartel DP: Mammalian microRNAs predominantly act to decrease target mRNA levels. Nature 466: 835-840, 2010

3. Xiao F, Yu J, Liu B, Guo Y, Li K, Deng J, Zhang J, Wang C, Chen S, Du Y, et al: A novel function of microRNA 130a-3p in hepatic insulin sensitivity and liver steatosis. Diabetes 63 : 2631-2642, 2014

4. Zhou B, Li C, Qi W, Zhang Y, Zhang F, Wu JX, Hu YN, Wu DM, Liu Y, Yan TT, et al: Downregulation of miR-181a upregulates sirtuin-1 (SIRT1) and improves hepatic insulin sensitivity. Diabetologia 55: 2032-2043, 2012.

5. Ferland-McCollough D, Ozanne SE, Siddle K, Willis AE and Bushell M: The involvement of microRNAs in Type 2 diabetes. Biochem Soc Trans 38: 1565-1570, 2010.

6. Gallagher IJ, Scheele C, Keller P, Nielsen AR, Remenyi J, Fischer CP, Roder K, Babraj J, Wahlestedt C, Hutvagner G, et al: Integration of microRNA changes in vivo identifies novel molecular features of muscle insulin resistance in type 2 diabetes Genome Med 2: 9, 2010.

7. Chen GQ, Lian WJ, Wang GM, Wang S, Yang YQ and Zhao ZW: Altered microRNA expression in skeletal muscle results from high-fat diet-induced insulin resistance in mice. Mol Med Rep 5: 1362-1368, 2012.

8. Zhang Y, Yang L, Gao YF, Fan ZM, Cai XY, Liu MY, Guo XR Gao CL and Xia ZK: MicroRNA-106b induces mitochondrial dysfunction and insulin resistance in $\mathrm{C} 2 \mathrm{C} 12$ myotubes by targeting mitofusin-2. Mol Cell Endocrinol 381: 230-240, 2013.
9. Zhang Y, Zhao YP, Gao YF, Fan ZM, Liu MY, Cai XY, Xia ZK and Gao CL: Silencing miR-106b improves palmitic acid-induced mitochondrial dysfunction and insulin resistance in skeletal myocytes. Mol Med Rep 11: 3834-3841, 2015.

10. Livak KJ and Schmittgen TD: Analysis of relative gene expression data using real-time quantitative PCR and the 2(-Delta Delta $\mathrm{C}(\mathrm{T})$ ) method. Methods 25: 402-408, 2001.

11. Liesa M, Palacin M and Zorzano A: Mitochondrial dynamics in mammalian health and disease. Physiol Rev 89: 799-845, 2009.

12. de Brito OM and Scorrano L: Mitofusin 2 tethers endoplasmic reticulum to mitochondria. Nature 456: 605-610, 2008.

13. Zorzano A: Regulation of mitofusin-2 expression in skeletal muscle. Appl Physiol Nutr Metab 34: 433-449, 2009.

14. Bach D, Naon D, Pich S, Soriano FX, Vega N, Rieusset J, Laville M, Guillet C, Boirie Y, Wallberg-Henriksson $\mathrm{H}$, et al: Expression of Mfn2, the Charcot-Marie-Tooth neuropathy type $2 \mathrm{~A}$ gene, in human skeletal muscle: Effects of type 2 diabetes, obesity, weight loss, and the regulatory role of tumor necrosis factor alpha and interleukin-6. Diabetes 54: 2685-2693, 2005.

15. Cartoni R, Léger B, Hock MB, Praz M, Crettenand A, Pich S, Ziltener JL, Luthi F, Dériaz O, Zorzano A, et al: Mitofusins 1/2 and ERRalpha expression are increased in human skeletal muscle after physical exercise. J Physiol 567: 349-358, 2005.

16. Nie Q, Wang C, Song G, Ma H, Kong D, Zhang X, Gan K and Tang Y: Mitofusin 2 deficiency leads to oxidative stress that contributes to insulin resistance in rat skeletal muscle cells. Mol Biol Rep 41: 6975-6983, 2014.

17. Zhang X, Wang C, Song G, Gan K, Kong D, Nie Q and Ren L: Mitofusion-2-mediated alleviation of insulin resistance in rats through reduction in lipid intermediate accumulation in skeletal muscle. J Biomed Sci 20: 45, 2013.

18. Borengasser SJ, Faske J, Kang P, Blackburn ML, Badger TM and Shankar K: In utero exposure to prepregnancy maternal obesity and postweaning high-fat diet impair regulators of mitochondrial dynamics in rat placenta and offspring. Physiol Genomics 46: 841-850, 2014. 